\title{
EDITORIAL
}

\section{¿ANTROPOENFERMERÍA O ENFERMEANTROPOLOGÍA?}

\author{
$M^{\text {a }}$ Antonia MARTORELL POVEDA \\ Enfermera y Antropóloga. Profesora Titular de Escuela Universitaria \\ Departamento de Enfermería, Universidad Rovira i Virgili \\ Avda. Roma, 17. 43005 Tarragona \\ mamp@eui.urv.es \\ 977251411
}

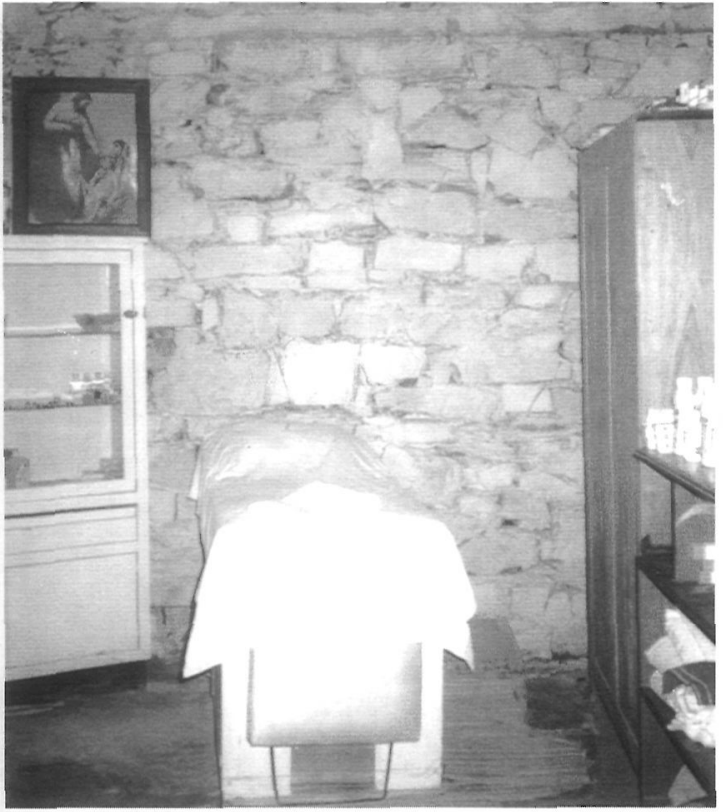

"ESTAR ALLI". Consultorio médico Huehuetla, (cabecera municipal) Puebla México.

\section{RESUMEN}

\section{¿ANTROPOLO-ENFERMERÍA O ENFREM- ANTROPOLOGÍA?}

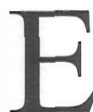

n el presente artículo, por un lado, se realiza una revisión sintética de las alianzas existentes entre la antropología y la enfermería y las posibles contribuciones de una disciplina a la otra. Por otro lado, desde un posicionamiento personal se intenta dar respuesta a la pregunta de ¿cómo llevar a cabo una investigación cuando el investigador trabaja en un campo clínico y tiene formación tanto en enfermería como en antropología? En cualquier caso, se resalta la relevancia metodológica que supone dar una respuesta a tal cuestión.

\section{ANTHROPO-NURSING OR NURSE-ANTH- ROPOLOGY?}

This article involves, on one hand, a synthetic review of existing links between anthropology and nursing and the potential contributions from one discipline to the other. On the other hand, from a personal point of view, an answer is sought to the questions: how to perform an investigation when the researcher works in a clinical area and has been trained in nursing as much as in anthropology? In any case, the methodological evidence of responding this question is remarked upon.

\section{ALIANZAS ENTRE ANTROPOLOGÍA Y ENFERMERÍA}

Según Holden y Littlewood (citados en Mulhall, A., 1996) puede hablarse de dos conceptos en los cuales se entremezclan la antropología y la enfermería y sobre los cuales es preciso hacer una distinción. El primero, se refiere a la antropología de la enfermería que incluye el estudio de las características socioculturales que definen la profesión enfermera; es decir, se centra en el estudio de las prácticas, tradiciones y rituales implicados en dicha disciplina. El segundo concepto denominado antropología para, o en, enfermería se interesa por la forma en que los conceptos y teorías de la antropología pue- 
den ser aplicados en la práctica de la enfermería; es decir, estudia cómo la antropología puede proporcionar un marco de trabajo para los cuidados.

Lo cierto es que entre enfermería y antropología existiría una larga historia de relación que nos llevaría incluso a hablar de una "alianza natural"' . Retomando parte de dicha historia (Dougherty, M. C. y Tripp-Réimer, T., 1985) vemos que, a principios del siglo XX, las enfermeras de Salud Pública ya mostraban un gran interés en el trabajo con grupos de inmigrantes. La labor de mejorar el entendimiento de diferentes culturas por parte de las enfermeras empezaría a quedar reflejada en varias publicaciones recogidas en la Public Health Nursing Quaterly. No obstante, la inclusión de la dimensión cultural en los cuidados de enfermería no empezará a ser clara hasta la década de los cuarenta. Dicho contenido fue especialmente introducido por aquellas enfermeras que servían con los militares durante la Segunda Guerra Mundial. Fue en dicho contexto donde empezaron a ver la necesidad de entender las diferencias culturales. A partir de aquí, la experiencia de estas enfermeras se trasladaría a las facultades de enfermería en donde enseñarían lo aprendido con los diferentes grupos étnicos.

En otro nivel, algunos antropólogos y científicos sociales fueron introduciendo el contenido cultural en la enseñanza de la enfermería. Fue particularmente importante la temprana contribución de la antropóloga Esther Lucille Brown. Brown, a la cabeza de la National Nursing Council, condujo un estudio sobre educación en enfermería que tuvo un gran impacto en la reforma educativa de esta disciplina. Poco a poco, la ciencia de la cultura gana importancia en la enfermería hasta que en 1937 la Liga Nacional de Enfermería (NLN) recomienda incluir un mínimo de diez horas semestrales de ciencias sociales en el currículum de los estudiantes, aspecto que también fue promovido por la Joint Commission. Distinguidas antropólogas como Margared Mead o Rhoda Metraux, a través de la Rusell Sage Foundation de la Cornell University School of Nursing también contribuyeron de forma importante en este proyecto.
En la década de los sesenta, muchas enfermeras americanas obtienen el grado de doctoras y varias de éstas lo hacen en el campo de la antropología. En 1968, en relación con la Sociedad Americana de Antropología Médica se constituye el Council on Nursing and Anthropology (CONRA), y a partir de aquí se sucederían otras organizaciones como la Transcultural Nursing Society, constituida en 1974, y la American Nurses' Association's Council on Inter-Cultural Diversity, creada en 1980. Finalmente, cabe señalar la gran contribución al desarrollo de la relación entre enfermería y antropología realizado por la enfermera-antropóloga Madeleine Leininger.

A partir de Leininger, la alianza entre antropología y enfermería se ha identificado en varias dimensiones. Foster (citado en Dougherty, M. C. y TrippRéimer, T., op. cit.) identifica cuatro niveles en los cuales se establecería la relación: a) los temas de la investigación, b) los enfoques conceptuales básicos de los problemas, c) la metodología de la investigación y, d) la identificación con los actores. Sin entrar en excesivos detalles vamos a ver más detenidamente cada uno de estos cuatro elementos.

En primer lugar, en cuanto a los temas de investigación, la antropología se ha interesado por creencias, valores y comportamientos relacionados con el proceso salud/enfermedad/atención. De forma similar, la práctica de enfermería se ha dirigido a conocer las respuestas y comportamientos de los sujetos ante las situaciones de salud/enfermedad/atención. No obstante, la enfermería difiere de la antropología en el nivel de análisis. Mientras la antropología se centraría prioritariamente en las normas culturales desde una perspectiva macro, la enfermería se concentraría en los individuos y utilizaría las normas culturales como elementos para entender los comportamientos del cliente. En la práctica enfermera, la valoración cultural incluye una comprensión de los valores, creencias y comportamientos del grupo de referencia de la persona y el ajuste de ésta al patrón normativo.

En segundo lugar, respecto a los métodos, la observación participante resulta central en ambas

'El concepto de "alianza natural" para referirse a las posibles relaciones y similitudes entre la antropología y la enfermería ha sido utilizado por varios autores. Así, por ejemplo, puede verse dicho uso en los artículos de Dougherty, M. L. y Tripp-Reimer, T. (1985) y de Mulhall, A. (1996). 
disciplinas. Las enfermeras, actúan como observadoras participantes activas tanto en el contexto domiciliario como hospitalario. A través de su proximidad física y su relación temporal con el sujeto aprendería los detalles más "íntimos" de la salud y la enfermedad. Así, como los antropólogos, las enfermeras, especialmente en el campo comunitario, accederían al entorno natural de los individuos y familias propiciándose el principio necesario y esencial del "estar allí". En el medio hospitalario, la situación es más difícil (Collière, M.F., 1993). En este caso, el profesional se encuentra en el medio institucional al que pertenece, siendo los enfermos y sus familiares los que acceden a ese medio. De todas formas, cada individuo llega con sus propios hábitos, sus creencias y su forma de entender el proceso salud/enfermedad. Así, como para el antropólogo que va al terreno, en cualquiera de los dos contextos citados, todo resultaría desconocido para la enfermera.

Con relación al tercer punto, tanto enfermería como antropología mostrarían un compromiso con el holismo. Más que otros profesionales de la salud, la enfermera se compromete al cuidado total del paciente, aspecto que resulta paralelo al interés antropológico por el género humano. Sin embargo, nuevamente, la diferencia de la aplicación holística se encontraría en el nivel de análisis.

Por último, con respecto al cuarto elemento señalado anteriormente, parecería claro que los antropólogos se identifican con las personas que estudian e investigan los problemas a partir de la perspectiva del actor ${ }^{2}$. Por su parte, las enfermeras también enfatizarían la empatía, el caring y la identificación con el sujeto, además de establecer una comunicación vinculada entre éste y el médico $u$ otros profesionales.

A partir de aquí, de acuerdo con Leininger (1964) parecería claro que aún cuando antropología y enfermería como disciplinas se muevan en órbitas separadas, los profesionales de ambos campos mantienen intereses similares dado que se preocupan por aspectos sociales y conductuales del estado de salud de los individuos. Además, cada una de las dos disciplinas tiene contribuciones específicas en la otra. Ya hemos visto más arriba de que modo la antropología ha contribuido al desarrollo de la enfermería y cómo esta última puede aprovechar la teoría y la investigación antropológica. Por su parte, la antropología se beneficia del entendimiento que las enfermeras tienen de los cuidados de salud. Además, la enfermería proporciona un terreno útil para la aplicación de la teoría de la antropología médica dado que la explicación e implementación de los métodos cualitativos ha sido la principal fuerza de los estudios de enfermería transcultural (Dougherty, M. C. y TrippRéimer, T., op. cit.).

Toda esta panorámica nos conduce a plantearnos alguna que otra pregunta, tal vez ya contestada (o no) por alguna enfermera, por alguna antropóloga o por alguna enfermera-antropóloga ${ }^{3}$. Así, por ejemplo, las cuestiones que nos planteamos son del tipo ¿cómo llevar a cabo la investigación cuando trabajamos en un campo clínico y tenemos educación en enfermería y en antropología?, ¿qué tipo de investigación debe ser la preferencial en este caso; es decir, hay que primar la investigación-acción o limitarse a una presentación de resultados?, ¿qué rol debe jugarse con los sujetos de la investigación: el de enfermera o el de antropóloga?, en cualquier caso, ¿pueden separarse ambos papeles o por el contrario deben suplementarse? De alguna manera, todos estos interrogantes, entre otros aspectos, pasarían por analizar las ventajas y los inconvenientes de la investigación en el campo sanitario cuando se tiene una doble formación como la sugerida.

De entrada, particularmente, me resulta difícil conseguir una separación estricta entre ambas disciplinas cuando estas forman parte de la formación de una misma persona, y más aún cuando, como hemos visto, entre las dos existe cierta complementariedad. Intentando ver las ventajas y los inconvenientes de esta situación creo que nos puede dar cierta luz a la hora de tomar nuestra decisión; una decisión que, en cualquier caso, considero de gran relevancia metodológica.

\footnotetext{
${ }^{2}$ Sobre el desarrollo de la aproximación metodológica centrada en el uso del punto de vista del actor en el campo de la Antropología Médica puede consultarse el trabajo de Menéndez, E. (1997)

${ }^{3}$ Utilizamos intencionadamente los términos en femenino para remarcar que a lo largo de la historia han sido las mujeres las que preferentemente han contribuido al desarrollo de la enfermería y a relacionar ésta con la antropología.
} 


\section{REFLEXIONES METODOLÓGICAS}

Ser enfermera, y como tal formar parte de un equipo, puede facilitar la entrada en un campo sanitario que deseamos estudiar. No obstante, del mismo modo, podría verse obstaculizada la tarea de investigador cuando los otros sólo ven a la persona que les va ha hacer la cura, que los acompaña en momentos críticos, habla con el médico sobre el dolor que uno siente, comparte el mismo turno o desayuna en la cafetería del personal.

El conocimiento que como profesional puede tener la enfermera sobre el terreno y su familiarización con el contexto sanitario no siempre actuaría en su beneficio. El hecho de participar en la misma dinámica que el resto de profesionales, o incluso que los pacientes, podría impedir desarrollar cierta curiosidad por aquello que parece resultar evidente. Es decir, se correría el riesgo de restar importancia a lo cotidiano, a lo rutinario. Sin embargo, cuando, como dice Van Dongen (1998), se accede al campo clínico como "antropóloga pura" no se necesita fomentar una curiosidad por lo autoevidente, todo resulta nuevo y extraño.
Esa misma familiarización con el contexto y las situaciones podría hacer suponer que ya no se es capaz de proporcionar información nueva. En este caso, sería necesario hacer un esfuerzo para entender un idioma que también utilizamos y compartimos. Asimismo, sería primordial adoptar una posición de relativa distancia para poder captar la multiplicidad de significados de palabras que para nosotras son totalmente normales y de uso habitual. Así, nos parece que la posición de la enfermera-antropóloga oscilaría en un movimiento constante entre, por un lado, la implicación y la empatía, y, por otro lado, entre la distancia necesaria para la reflexión y la escritura.

Así, una vez más, se resaltaría la importancia de actuar, el máximo posible, como un extraño que se sorprende por todo lo que sucede a su alrededor. Con esta actitud, la enfermera-antropóloga sería capaz de descubrir nuevos idiomas, nuevos significados, situaciones de conflicto, etc. No obstante, por lo mismo que señalábamos un poco más arriba, puede resultar algo difícil que los demás vean a la enfermera como alguien ajeno al lugar



La curiosidad por el "EXTRAÑO". Centro de Salud. Cominidad Cinco de Mayo. Huehuetla, Puebla, México.

" La situación que vivía la enfermera-antropóloga no solo estaría determinada por su papel en el terreno sino también por el propio objeto de estudio. Es decir, entiendo que la situación no sería la misma si se estudia, por ejemplo, la vivencia de un trastorno concreto $o$ se pretende realizar una investigación sobre la institución. 
cuando están compartiendo espacios y situaciones con ella. Por este motivo, creemos que es fundamental la necesidad de clarificar desde el inicio de la investigación cuál va ha ser nuestra postura, qué vamos a hacer y cómo lo vamos a hacer.

En principio, parece que la vivencia del "estar allí" quedaría resuelta por la propia situación laboral de la enfermera. No obstante, ésta tendrá que ser capaz de saber encontrar el equilibrio entre la implicación y la distancia. Por parte de la profesional de enfermería, considero que debería existir cierto nivel de implicación, pero una implicación y empatía que al mismo tiempo le permita mantener el distanciamiento necesario para la reflexión y el análisis de la realidad. Así, desde un principio, deberíamos reflexionar sobre nuestro grado de participación y complicidad tanto como investigador y como profesional. La posición que a este respecto adoptemos influiría directamente en la información que recojamos. Es necesario pensar que un mayor énfasis en la parte profesional podría influir, por ejemplo, en los datos que nos facilitasen los propios compañeros, bien limitándolos o bien siendo presentados con poca claridad.

Pero la cuestión no es sólo "estar allí". Hay que hablar, entrevistar, conocer la vida de las personas, consultar archivos, leer notas de evolución clínica, etc. Nuevamente, la posición "privilegiada" de la enfermera facilitaría el acceso a esta información. No obstante, a partir de aquí se requeriría tomar una decisión inicial sobre cómo organizar el trabajo de campo. Es decir, ¿se trataría de trabajar como profesional y al mismo tiempo realizar la observación y la recolección de información? o, por el contrario, ¿sería preciso programarse los momentos que se dedican específicamente a la investigación? Por las dificultades que ya hemos ido exponiendo que pueden presentarse a la hora de combinar ambos papeles, en este caso, considero más oportuno establecer una programación del tiempo que se dedica a cada situación. Pienso que a su vez esto puede contrarrestar algunos de los riesgos ya expuestos y clarificar aún más nuestro papel como investigador.

Finalmente, otro dilema se nos puede plantear a la hora de escribir y presentar los resultados de la investigación; sobre todo cuando nuestra etnografía ha tenido por objeto de estudio la institución en la que trabajamos o los compañeros con los cuales compartimos la actividad profesional. En cualquier caso se ha de procurar escribir con un lenguaje los más entendible posible, y aún mas cuando esperamos que nuestra investigación comporte cambios.

Como hemos visto, las similitudes entre enfermería y antropología serían notorias, tanto en el ámbito de métodos como de enfoques conceptuales. En mi opinión, es importante que las enfermeras hayan aprendido (y estén aprendiendo) a utilizar la antropología en su propio campo profesional, dando a los cuidados una mayor dimensión cultural; máxime si tenemos en cuenta que actualmente los flujos migratorios -derivados en buena parte del proceso de globalización- plantean nuevas situaciones sobre el proceso salud/enfermedad/atención a las que la enfermera debe saber dar respuesta. En este ámbito parece fundamental el papel de traductora cultural que la enfermeraantropóloga puede ejercer en relación con dicho proceso. Así, de acuerdo con Mulhall (op. cit.), el sentimiento de proporcionar cuidados culturalmente apropiados sería muy loable, pero hablar de los otros o clamar la solidaridad con ellos es una gran responsabilidad que los profesionales debemos reflejar constantemente. En este sentido, pienso que la formación en antropología puede resultar muy beneficiosa para la práctica enfermera.

\section{BIBLIOGRAFÍA}

COLLiėRE, M.F. (1993) Utilización de la antropología para abordar las situaciones de cuidados. Revista rol de Enfermería 179-180, 71-80.

DOUGHERTY, M.C. Y TRIPP-RÉIMER, T. (1985) The interface of nursing and anthropology. Ann. Rev. Anthropol. 14, 219-241.

LEINNINGER, M. (1964) Nursing and anthropology: two worlds to blend. Greyden Press, Columbus OH, pp. $17-44$.

MENENDEZ, E.L. (1997) Estructura social y estructura de significado: el punto de vista del actor. En Gonzalez Reboredo, X. M. (comp.), Medicina popular en anrtopologia Saude. Actas del Simposio Internacional en homenaxe a D. Antonio Fraguas. Consello de Cultura Gallega, Santiago de Compostela, pp. 281305.

Mulhall, A. (1996) Anthropology, nursing and midwifery: a natural alliance? Int. J. Nurs. Stud. 33 (6), 629-637.

VAN Dongen, E. (1998) Strangers on terra cognita: authors of the Other in a mental hospital. Anthropology and Medicine 5 (3), 279-293. 